\title{
A discussion on finite-difference schemes for low Prandtl number Rayleigh-Bénard convection
}

\author{
X. Luo \& W.-K. Chen \\ Institute of Thermal Engineering, University of Shanghai for Science and \\ Technology, People's Republic of China
}

Dedicated to Prof. Dr.-Ing. Wilfried Roetzel on the occasion of his $70^{\text {th }}$ birthday

\begin{abstract}
The natural convection in a horizontal fluid layer heated from below has complex dynamic behaviour. For the Rayleigh-Bénard convection of low Prandtl number fluids, the calculated flow and temperature fields are very sensitive to the truncation error of numerical algorithms. Different kinds of finite-difference schemes might yield different numerical results. In the present work the error analysis of the upwind scheme and QUICK scheme for the Rayleigh-Bénard convection of low Prandtl number fluid was conducted. It shows that the upwind scheme will introduce numerical dispersion. This effect enlarges the viscosity term of the momentum equations and therefore no oscillation could be predicted. The QUICK scheme has higher calculation accuracy. However, it introduces an additional third-order differential term which might overestimate the oscillation effect.

Keywords: Rayleigh-Bénard convection, low Prandtl number fluid, two-dimensional roll, finite-difference scheme, QUICK scheme.
\end{abstract}

\section{Introduction}

The natural convection in a horizontal layer confined by two rigid boundaries and heated from below is well known as Rayleigh-Bénard convection. This phenomenon reveals series non-linear characteristics and complex dynamic behaviour and has been well investigated [1-2]. The studies of low Prandtl 
number Rayleigh-Bénard convection have been motivated not only by its astrophysical applications but also by its special flow and heat transfer characteristics because in this case the non-linear inertial terms become significant. Clever and Busse [3] used the Galerkin technique to obtain the Nusselt number for low Prandtl number convection at various Rayleigh numbers. According to the linear stability analysis, the critical Rayleigh number $R a_{c}$ and critical wave number $k_{c}$ for two rigid boundaries are given as $R a_{c}=1707.762$ and $k_{c}=3.117$, respectively [1]. Bertin and Ozoe [4] calculated the problem with a finite-element method and found that $R a_{c}$ increases with the decrease of $\mathrm{Pr}$. The computed critical Rayleigh number for $\operatorname{Pr}=0.01$ and $k=3.14$ is given as $R a_{c}=2095.0$. Later Ozoe et al. [5] used a more accurate second-order central difference scheme to solve the problem and the calculated Nusselt number agreed closely with those of Clever and Busse [3]. The computed value of $R a_{c}$ for all values of $\mathrm{Pr}$ also agreed well with the theoretical value. Their results show that the Rayleigh-Bénard convection of low Prandtl number fluid is very sensitive to the numerical algorithm.

As the Rayleigh number is larger than its critical value, the fluid begins to move and forms a steady flow pattern of two-dimensional rolls parallel to each other. For higher Rayleigh number the rolls become unsteady and the bending of the rolls propagates along the roll axis in time. By means of stability analysis for steady convection rolls Clever and Busse [6] discussed the oscillation instability and the critical Rayleigh number $R a_{t}$ for the onset of oscillation. Later they found that the transition from thermal convection in the form of rolls in a fluid layer heated from below to travelling-wave convection occurs at $R a_{t}=1854$ in the limit of low Prandtl numbers and in the presence of rigid boundaries [7]. Ozoe and Hara [8] carried out numerical computations with a second-order central difference approximation to predict $R a_{t}$. The computing region is 4 times as large as the height, i.e., the aspect ratio $A=4$. For the grid size of 0.02 , the oscillation occurs at $R a_{t} \approx 4500$ for 4-roll pattern and $P r=0.01$. By extrapolation to zero grid size they inferred that the critical oscillatory Rayleigh number $R a_{t}$ was less than 2000. Yang et al. [9] and Wang et al. [10] used the SIMPLE algorithm with QUICK (quadratic upwind interpolation of convective kinematics) scheme to solve the same problem as that of Ozoe and Hara [8] with $A=4$ and $\operatorname{Pr}=0.01$ and found that the fluid flow and heat transfer is steady and stable for $R a \leq 2200$. Their numerical calculation showed that the oscillation occurs at $R a=2500$. As has been mentioned by Yang et al. [11], for low Prandtl number fluid the calculated Nusselt numbers with different numerical schemes are quite different. They suggested a possible explanation that the problem might have bifurcations and the results from different numerical schemes might lie at different branches of the solution.

In the present work, this problem was solved numerically with the upwind scheme, power law scheme and QUICK scheme. The Taylor series expansion was used to analyse the truncation errors of the schemes. It was found that the upwind scheme would introduce fictitious viscosity and underestimate the value of Nusselt number. The QUICK scheme was more suitable for the low Prandtl 
number natural convection. However, it introduces a third-order differential term which might affect the oscillation characteristics of the physical model.

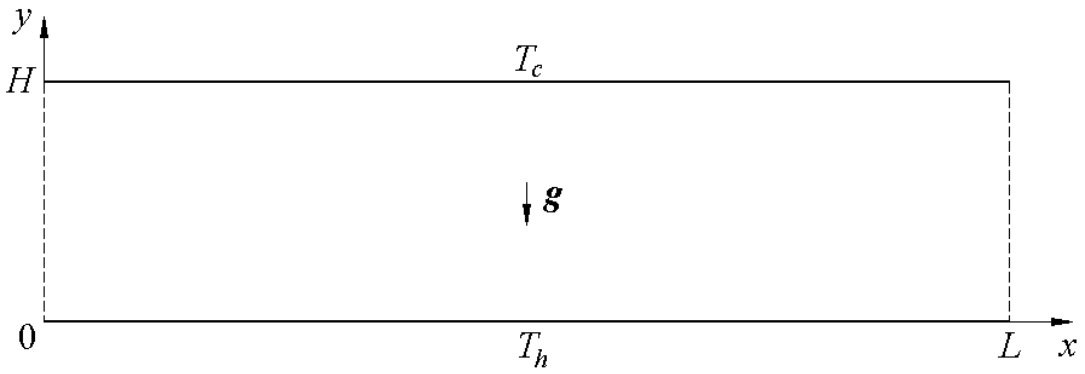

Figure 1: Schematic diagram of the horizontal fluid layer heated from below.

\section{Mathematical model}

Let us consider a horizontal fluid layer with two rigid boundaries at its upper and lower surfaces, as shown in fig. 1. The fluid layer is heated from below and cooled from above. The temperatures at the upper and lower rigid boundaries are $T_{h}$ and $T_{c}$, respectively, and $T_{h}>T_{c}$. The fluid expands when it is heated, therefore it suffers an upward buoyancy force. The problem is simplified with the following assumptions: (1) The flow pattern is assumed to be two-dimensional; (2) All properties of the fluid are constant except the density in the buoyancy term of the momentum equation which is a linear function of the fluid temperature; (3) The left and right boundaries are two symmetric boundaries, that means that the wave number in this model is fixed.

The following dimensionless variables and parameters are used for the modeling:

$$
\begin{gathered}
X=x / H, Y=y / H, \tau=U_{R} t / H, U=u / U_{R}, V=v / U_{R}, A=L / H, \\
U_{R}=\frac{a}{H} \sqrt{R a P r}, D_{V}=\sqrt{\frac{P r}{R a}}, D_{T}=\frac{1}{\sqrt{R a P r}}, \operatorname{Pr}=\frac{v}{a}, R a=\frac{g \beta\left(T_{h}-T_{c}\right) H^{3}}{v a} .
\end{gathered}
$$

We assume that at first there is no motion in the fluid, and the temperature distribution is in a steady state. The steady-state temperature and reduced pressure distributions can be expressed as

$$
T_{\tau=0}=T_{c}+\left(T_{h}-T_{c}\right)(1-Y)
$$

and

$$
p_{\tau=0}=\rho U_{R}^{2} Y(1-Y / 2) .
$$


It is convenient to introduce the dimensionless temperature and pressure perturbations,

$$
\begin{gathered}
\theta=\frac{T-T_{\tau=0}}{T_{h}-T_{c}}=\frac{T-T_{c}}{T_{h}-T_{c}}-(1-Y), \\
P=\frac{p-p_{\tau=0}}{\rho U_{R}^{2}}=\frac{p}{\rho U_{R}^{2}}-Y\left(1-\frac{Y}{2}\right) .
\end{gathered}
$$

The dimensionless governing equation system can then be written as:

$$
\begin{gathered}
\frac{\partial U}{\partial \tau}+U \frac{\partial U}{\partial X}+V \frac{\partial U}{\partial Y}=-\frac{\partial P}{\partial X}+D_{V}\left(\frac{\partial^{2} U}{\partial X^{2}}+\frac{\partial^{2} U}{\partial Y^{2}}\right) \\
\frac{\partial V}{\partial \tau}+U \frac{\partial V}{\partial X}+V \frac{\partial V}{\partial Y}=-\frac{\partial P}{\partial Y}+D_{V}\left(\frac{\partial^{2} V}{\partial X^{2}}+\frac{\partial^{2} V}{\partial Y^{2}}\right)+\theta \\
\frac{\partial \theta}{\partial \tau}+U \frac{\partial \theta}{\partial X}+V \frac{\partial \theta}{\partial Y}=D_{T}\left(\frac{\partial^{2} \theta}{\partial X^{2}}+\frac{\partial^{2} \theta}{\partial Y^{2}}\right)+V \\
\frac{\partial U}{\partial X}+\frac{\partial V}{\partial Y}=0
\end{gathered}
$$

with the initial and boundary conditions

$$
\begin{array}{ll}
\tau=0: & \theta=P=0 ; \\
X=0 \text { and } X=A: & U=\frac{\partial V}{\partial X}=\frac{\partial \theta}{\partial X}=0 ; \\
Y=0 \text { and } Y=1: & U=V=\theta=0 .
\end{array}
$$

The average Nusselt number is calculated at the lower plate,

$$
N u=1-\left.\frac{1}{A} \int_{0}^{A} \frac{\partial \theta}{\partial Y}\right|_{Y=0} d X
$$

The zero solution of the governing equation system (5)-(11) corresponds to the motionless state which is stable for $R a<R a_{c}$. Even the fluid is stirred before, the disturbance will gradually vanish and finally the fluid approaches the steady state again. However, If $R a>R a_{c}$, this zero solution becomes unstable. Any 
small disturbance will be enlarged gradually and finally the Rayleigh-Bénard convection will be set up. For small value of $R a-R a_{c}$, the motion is twodimensional. With the increase of the Rayleigh number, the flow becomes steady three-dimensional flow, unsteady flow, and finally, turbulent flow [12].

In this paper eqns. (5)-(11) for $R a>R a_{c}$ were solved with finite-difference methods. The research was emphasised on the oscillatory characteristics of the two-dimensional rolls, which reflects some special features of the upwind scheme and QUICK scheme.

\section{Comparison of finite-different schemes}

Ozoe et al. [5] used the vorticity and stream function to eliminate the pressure gradient terms in eqns. (5)-(11) and solved the problem by central differences for the derivatives in space and alternating-direction implicit differences for the derivatives in time. They calculated the Nusselt number for Ra $\leq 3000$ with the aspect ratio $A=1$. They found that the grid size had significant effect on the calculated Nusselt number and had to extrapolate their results to the case of $(\Delta X$, $\Delta Y) \rightarrow 0$. As shown in table 1 , their results agree well with those of Clever and Busse [3] obtained by Galerkin method. No oscillation is found in their calculation.

Table 1: The calculated Nusselt numbers from different sources.

\begin{tabular}{|c|c|c|c|c|c|}
\hline \multirow{3}{*}{$R a$} & \multicolumn{5}{|c|}{$\begin{array}{c}N u \\
(\operatorname{Pr}=0.01)\end{array}$} \\
\hline & $\begin{array}{c}\text { Clever and } \\
\text { Busse [3] }\end{array}$ & $\begin{array}{l}\text { Ozoe and } \\
\text { Ukeba [5] }\end{array}$ & $\begin{array}{c}\text { Ozoe and } \\
\text { Hara [8] }\end{array}$ & $\begin{array}{l}\text { Yang } \\
\text { Wan }\end{array}$ & $\begin{array}{l}\text {. [9] and } \\
\text { al. [10] }\end{array}$ \\
\hline & $k=3.11$ & $\begin{array}{c}k=3.14 \\
(1 \text { roll })\end{array}$ & $\begin{array}{l}k=3.14 \\
(4 \text { rolls })\end{array}$ & $\begin{array}{l}k=3.14 \\
(4 \text { rolls })\end{array}$ & $\begin{array}{l}k=3.93 \\
(5 \text { rolls })\end{array}$ \\
\hline 2000 & 1.01955 & & & 1.018 & \\
\hline 2300 & & $1.105^{* *}$ & & 1.094 & 1.104 \\
\hline 2500 & 1.17335 & $1.18^{* *}$ & & $1.167^{*}$ & $1.176^{*}$ \\
\hline 3000 & 1.33978 & $1.35^{* *}$ & & $1.269^{*}$ & $1.355^{*}$ \\
\hline 4000 & 1.59614 & & & $1.511^{*}$ & $1.609^{*}$ \\
\hline 5000 & & & 1.259 & $1.651^{*}$ & $1.793^{*}$ \\
\hline 6000 & 1.89397 & & $1.289^{*}$ & $1.764^{*}$ & $1.933^{*}$ \\
\hline 10000 & 2.22264 & & $1.502^{*}$ & $2.052^{*}$ & $2.316^{*}$ \\
\hline
\end{tabular}

(*: The solution is oscillating; **: Data are extrapolated to zero grid size.)

Ozoe and Hara [8] further calculated the Nusselt number for higher Rayleigh numbers, $4000 \leq R a \leq 2.8 \times 10^{5}$. The data listed in table 1 are taken from fig. 2 of [8]. These data are calculated under the grid size of 0.02 . Their 
calculation shows that the oscillation appears when $R a \geq 6000$. However, their predicted values of $\mathrm{Nu}$ are much lower than those of Clever and Busse [3].

We calculated the problem by the SIMPLE algorithm [13] and upwind scheme with $\operatorname{Ra}=5000, \operatorname{Pr}=0.01, A=4$ and found that the grid size of 0.02 would yield $N u=1.29$ which is a little higher than that of Ozoe and Hara [8]. This value is not correct because the grid size of 0.01 would yield another value of $N u=1.46$. No oscillation can be found in the numerical solutions. The power law scheme yields the same results as the upwind scheme. Applying the Taylor series expansion to the upwind scheme, we can find that the upwind scheme introduces an additional dimensionless viscosity $U \Delta X / 2$. In the above example, because of the low Prandtl number, we have $D_{V}=1.414 \times 10^{-3}$. The maximum dimensionless velocity $U_{\max }=0.7776$. Thus, for $\Delta X=0.02$, this fictitious viscosity could be 5.5 times as large as the real viscosity and therefore leads to a lower value of $\mathrm{Nu}$.

Yang et al. [11] compared the QUICK scheme with the power law scheme and found that for natural convection of low Prandtl number fluids the QUICK scheme provides higher accuracy. Therefore, the QUICK scheme was used in the calculations of Yang et al. [9] and Wang et al. [10]. Some results for $\mathrm{Pr}=0.01$, $A=4$ and $\Delta X=\Delta Y=0.02$ are shown in table 1 . The calculated values of $N u$ for 4 rolls are close to those of Clever and Busse [3].

Applying the Taylor series expansion to the QUICK scheme,

$$
\begin{aligned}
& \frac{\phi(X, \tau)-\phi(X, \tau-\Delta \tau)}{\Delta \tau}+\frac{U}{\Delta X}\left[\frac{3}{8} \phi(X, \tau)+\frac{3}{8} \phi(X+\Delta X, \tau)-\frac{7}{8} \phi(X-\Delta X, \tau)\right. \\
& \left.+\frac{1}{8} \phi(X-2 \Delta X, \tau)\right]=D \frac{\phi(X+\Delta X, \tau)+\phi(X-\Delta X, \tau)-2 \phi(X, \tau)}{\Delta X^{2}},
\end{aligned}
$$

we have,

$$
\frac{\partial \phi}{\partial \tau}+\mathrm{O}(\Delta \tau)+U \frac{\partial \phi}{\partial X}=D \frac{\partial^{2} \phi}{\partial X^{2}}+R\left(\Delta X^{2}\right),
$$

in which

$$
R\left(\Delta X^{2}\right)=\frac{\Delta X^{2}}{24}\left(D \frac{\partial^{4} \phi}{\partial X^{4}}-U \frac{\partial^{3} \phi}{\partial X^{3}}\right)+\mathrm{O}\left(\Delta X^{3}\right) .
$$

It shows that the QUICK scheme does not introduce the fictitious viscosity term and has the truncation error of $\mathrm{O}\left(\Delta X^{2}\right)$, therefore the predicted $N u$ values are more accurate.

Wang et al. [10] also used the QUICK scheme to predict the roll oscillation. They found that for $P r=0.01, A=4$ and $\Delta X=\Delta Y=0.02$ the oscillation occurs at $R a=2500$, which is much higher than the theoretical value of the critical 
oscillatory Rayleigh number, $R a_{t}=1854$. However, they neither tested the effect of the grid size on the oscillation characteristics nor analysed the stability characteristics of the QUICK scheme.

For low Prandtl number fluid, we have $D / U \sim 10^{-2}$. Thus, eqn. (15) is simplified as,

$$
R\left(\Delta X^{2}\right)=-\frac{U \Delta X^{2}}{24} \frac{\partial^{3} \phi}{\partial X^{3}}+\mathrm{O}\left(\Delta X^{3}\right) .
$$

In such a case, the QUICK scheme will introduce an additional term into the governing equations which affects the oscillation characteristics of the RayleighBénard convection. In fact, the stability of the QUICK scheme is conditional. The stability condition is given as [14],

$$
P_{\Delta}=\frac{U \Delta X}{D} \leq \frac{8}{3}
$$

For $R a=2500, P r=0.01$ and $\Delta X=0.02$, the maximum dimensionless velocity $U_{\max }=0.867$, which yields $P_{\Delta}=8.67$ and the condition (17) can no longer be valid. Although an oscillation solution can be obtained with the QUICK scheme, but it is not sure whether this oscillation is caused by the instability of the numerical scheme or by the characteristics of the physical problem or by both of them.

To verify the numerical schemes, two critical values should be considered. One is the critical oscillatory Rayleigh number $R a_{t}$, which is difficult to be determined with direct numerical simulation. By extrapolation to zero grid size, Ozoe and Hara [8] estimated that $R a_{t}<2000$, which is near to the theoretical value, $R a_{t}=1854$. We tested a calculation with QUICK scheme for $R a=2000$, $\operatorname{Pr}=0.01$ and $\Delta X=0.005$. And contrary to our expectation, after several thousand hours of computing time of a PC with a $2.8 \mathrm{GHz} \mathrm{CPU}$, we obtained a steady convection (The relative velocity disturbance $\Delta U / U_{\max }$ was less than $10^{-9}$ ). When we added a small disturbance in Rayleigh number, an oscillatory disturbance was set up. The disturbance of the maximum vertical velocity component is shown in fig. 2. At $\tau=30$, there is some change in the frequency. After that point the oscillation becomes violent. Then, at about $\tau=55$, we increased the iteration accuracy and set the minimum iteration times to 3 . And the oscillation gradually vanished. The solution reached the steady convection again.

The other criterion is the oscillation frequency. According to fig. 12 of Clever and Busse [6], the theoretical frequency of the oscillatory disturbance for $\operatorname{Pr}=0.01$ and $R a=2500$ is about 0.01 (after the conversion between different definitions of dimensionless time). But the QUICK scheme of Yang et al. [11] with the grid size of 0.02 resulted a frequency of 0.3125 (see fig. 2 of [11]). Our calculation with the QUICK scheme gave a frequency of 0.3344 . Both of them are much larger than the theoretical value given by Clever and Busse [6]. 


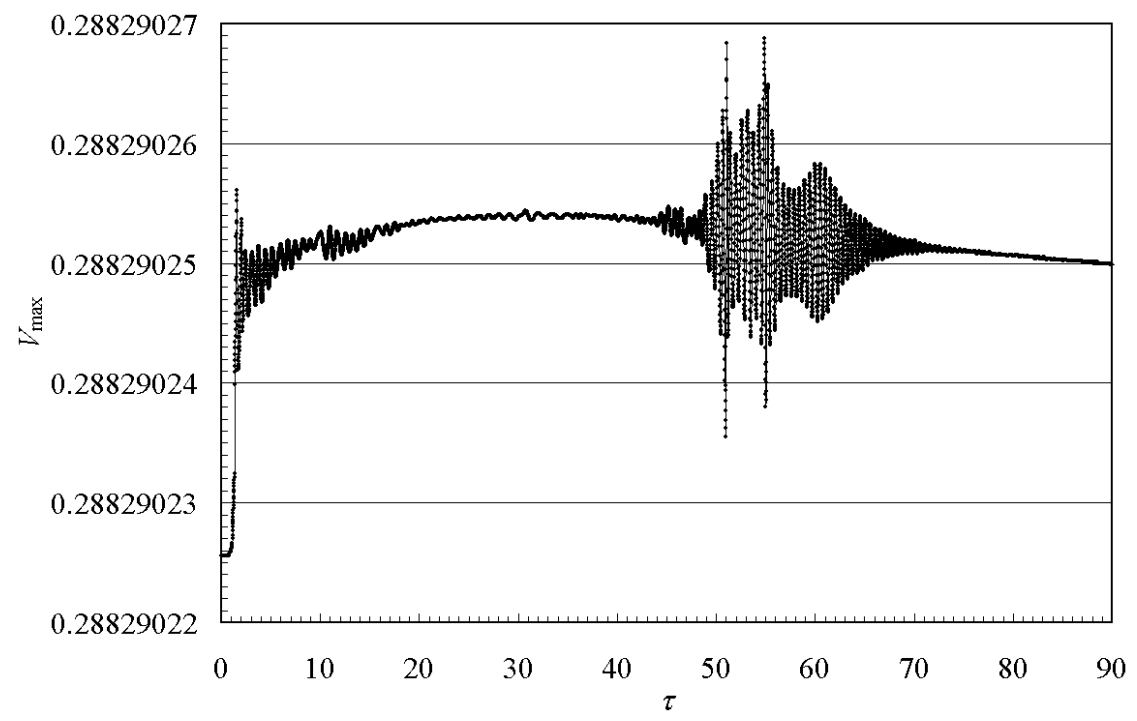

Figure 2: Velocity disturbance due to a disturbance in Rayleigh number ( $R a=2000, \operatorname{Pr}=0.01, A=4$ and $\Delta X=0.005)$.

These examples show that the Rayleigh-Bénard convection of low Prandtl number fluid is very sensitive to the oscillation characteristics of the finitedifference schemes. Therefore, more accurate schemes should be developed for the direct simulation of such a problem.

\section{Conclusions}

The Rayleigh-Bénard convection of low Prandtl number fluid is difficult to be solved because of its nonlinear properties and special dynamic characteristics. The two-dimensional transient numerical calculations with SIMPLE algorithm were carried out to simulate such a problem. Different numerical schemes such as upwind scheme, power law scheme and QUICK scheme were used in the calculation. We find that the upwind scheme and power law scheme are not suitable for the direct simulation of low Prandtl number Rayleigh-Bénard convection because they will introduce a fictitious dispersion term. The QUICK scheme provides an accuracy of the second order and will not introduce the numerical dispersion into the problem to be solved. The predicted values of Nusselt number are reasonably accurate. However, it fails to predict the critical oscillatory Rayleigh number and the oscillation frequency because of the stability and oscillation characteristics of the QUICK scheme itself. New numerical schemes should be developed to simulate the low Prandtl number Rayleigh-Bénard convection.

In the present work we have restricted our calculation with a fixed aspect ratio $A=4$, i.e., the wave number $k=3.14$. In fact, the critical Rayleigh numbers also depend on the wave number $k$ and the number of rolls to be considered. 
Therefore, there remains a lot of unsolved problems in the numerical simulation of the Rayleigh-Bénard convection.

\section{Acknowledgements}

The present research was sponsored by the National Natural Science Foundation of China (No. 50478113), Shanghai Leading Academic Discipline Project (No. T0503) and Shanghai Pujiang Program (No. 05PJ14078).

\section{Nomenclature}

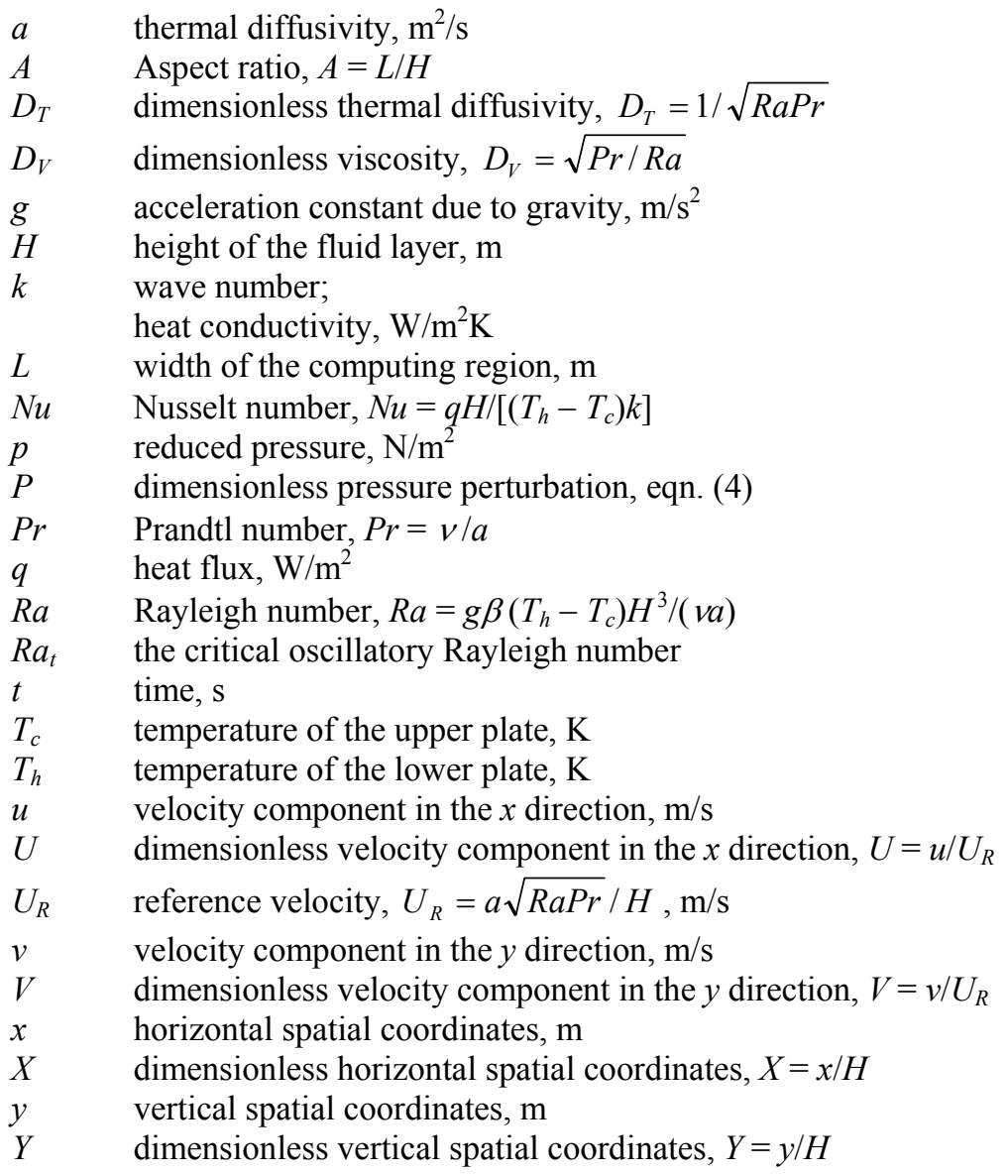

\section{Greek symbols}

$\beta \quad$ volumetric coefficient of expansion, $1 / \mathrm{K}$

$v \quad$ kinematic viscosity, $\mathrm{m}^{2} / \mathrm{s}$

$\theta \quad$ dimensionless temperature perturbation, eqn. (3)

$\tau \quad$ dimensionless time, $\tau=U_{R} t / H$

\section{Subscript}

$c \quad$ critical value 


\section{References}

[1] Getling, A.V., Rayleigh-Benard Convection-Structures and Dynamics, World Scientific: Singapore, 1998.

[2] Koschmieder, E.L., Benard Cells and Taylor Vortices, Cambridge University Press, 1993.

[3] Clever, R.M. \& Busse, F.H., Low-Prandtl-number convection in a layer heated from below, Journal of Fluid Mechanics, 102, 61-74, 1981.

[4] Bertin, H. \& Ozoe, H., Numerical study of two-dimensional natural convection in a horizontal fluid layer heated from below, by finite-element method: influence of Prandtl number, International Journal of Heat and Mass Transfer, 29(3), 439-449, 1986.

[5] Ozoe, H., Ukeba, H. \& Churchill, S.W., Numerical analysis of natural convection of low Prandtl number fluids heated from below, Numerical Heat Transfer, Part A, 26, 363-374, 1994.

[6] Clever, R.M. \& Busse, F.H., Transition to time-dependent convection, Journal of Fluid Mechanics, 65, part 4, 625-645, 1974.

[7] Clever, R.M. \& Busse, F.H., Convection at very low Prandtl numbers, Physics of Fluids, Series A, 2(3), 334-339, 1990.

[8] Ozoe, H. \& Hara, T., Numerical analysis for oscillatory natural convection of low Prandtl number fluid heated from below, Numerical Heat Transfer, Part A, 27, 307-317, 1995.

[9] Yang, M., Chui, X.-Y., Tao, W.-Q. \& Ozoe, H., Bifurcation and oscillation of natural convection in a horizontal layer of low Prandtl number fluid, Journal of Engineering Thermophysics, 21(4), 461-465, 2000 (in Chinese).

[10] Wang, J.-G., Yang, M., Zhao, M., Cui, X.-Y. \& Zhang, L.-X., Bifurcation of natural convection for low Prandtl number fluid heated from below, Journal of Engineering Thermophysics, 24(1), 76-78, 2003 (in Chinese).

[11] Yang, M., Li, X.-H., Tao, W.-Q. \& Ozoe, H., Computation and Comparison for heat and flow using a QUICK and other difference schemes, Journal of Engineering Thermophysics, 20(5), 593-597, 1999 (in Chinese).

[12] Krishnamurti, R., Some further studies on the transition to turbulent convection, Journal of Fluid Mechanics, 60(3), 285-303, 1973.

[13] Patankar, S.V., Numerical Heat Transfer and Fluid Flow, Hemisphere: New York, 1980.

[14] Tao, W.-Q., Numerical Heat Transfer, Xi'an Jiaotong University Press: Xi'an, pp. 220-231, 1988 (in Chinese). 